ARTICLE

Received 25 Aug 2016 | Accepted 10 Nov 2016 | Published 21 Dec $2016 \quad$ DOl: 10.1038/ncomms13902

\title{
Folding of xylan onto cellulose fibrils in plant cell walls revealed by solid-state NMR
}

\author{
Thomas J. Simmons ${ }^{1, \star}$, Jenny C. Mortimer ${ }^{1, *, \dagger}$, Oigres D. Bernardinelli ${ }^{1,2,3, \dagger}$, Ann-Christin Pöppler ${ }^{3}$, \\ Steven P. Brown ${ }^{3}$, Eduardo R. deAzevedo ${ }^{2}$, Ray Dupree ${ }^{3, \star \star} \&$ Paul Dupree ${ }^{1, \star \star}$
}

Exploitation of plant lignocellulosic biomass is hampered by our ignorance of the molecular basis for its properties such as strength and digestibility. Xylan, the most prevalent non-cellulosic polysaccharide, binds to cellulose microfibrils. The nature of this interaction remains unclear, despite its importance. Here we show that the majority of xylan, which forms a threefold helical screw in solution, flattens into a twofold helical screw ribbon to bind intimately to cellulose microfibrils in the cell wall. ${ }^{13} \mathrm{C}$ solid-state magic-angle spinning (MAS) nuclear magnetic resonance (NMR) spectroscopy, supported by in silico predictions of chemical shifts, shows both two- and threefold screw xylan conformations are present in fresh Arabidopsis stems. The twofold screw xylan is spatially close to cellulose, and has similar rigidity to the cellulose microfibrils, but reverts to the threefold screw conformation in the cellulose-deficient irx3 mutant. The discovery that induced polysaccharide conformation underlies cell wall assembly provides new principles to understand biomass properties.

\footnotetext{
${ }^{1}$ Department of Biochemistry and Leverhulme Centre for Natural Material Innovation, Hopkins Building, Downing Site, University of Cambridge, Cambridge CB2 1QW, UK. ${ }^{2}$ Instituto de Física de São Carlos, Departamento de Física e Ciência Interdisciplinar, Universidade de São Paulo, Caixa Postal 369, São Carlos, Sao needs, São Paulo 13660-970, Brazil. ${ }^{3}$ Department of Physics, University of Warwick, Coventry CV4 7AL, UK. ${ }^{*}$ These authors contributed equally to this work. ${ }^{\star \star}$ These authors jointly supervised this work. † Present addresses: Joint Bioenergy Institute, Lawrence Berkeley National Lab, Berkeley, California 94720 , USA (J.C.M); Instituto de Química, Universidade Estadual de Campinas, Caixa Postal 6154, CEP 13084-862, Campinas, Brazil (O.D.B). Correspondence and requests for materials should be addressed to R.D. (email: ray.dupree@warwick.ac.uk) or to P. D. (email: pd101@cam.ac.uk).
} 
P lant secondary cell walls are strong composites of polysaccharides, lignin and proteins that are crucial for plant structure. They provide the most abundant renewable materials on Earth. This lignocellulosic material is therefore the focus of biofuel development ${ }^{1}$, and wood is widely used for many industries such as building construction, paper and packaging. Polysaccharides constitute over $70 \%$ of dry cell wall lignocellulosic biomass and can be categorized as cellulose, hemicelluloses and pectins in decreasing order of prevalence (Supplementary Fig. 1) ${ }^{2,3}$. Properties such as biomass recalcitrance and timber strength arise from secondary cell wall molecular architecture: the molecular arrangements and conformations of the polysaccharides and other components within the secondary cell wall. Nevertheless, cell wall molecular architecture is very poorly understood.

The semi-crystalline cellulose microfibrils provide the best understood example of molecular architecture in lignocellulose. Its component $\beta$ - $(1 \rightarrow 4)$-D-glucan chains fold in a twofold helical screw conformation (one $360^{\circ}$ twist per 2 glycosidic bonds). The pattern of hydrogen bonding interactions is well understood from X-ray diffraction and magic-angle spinning (MAS) solid-state nuclear magnetic resonance (NMR) studies ${ }^{4-6}$. On the other hand, the intermolecular interactions that occur between cellulose fibrils and hemicelluloses have been a matter of debate for several decades ${ }^{2,7}$. Xyloglucan was thought to crosslink microfibrils in primary cell walls. Multidimensional MAS NMR analyses of primary cell walls, pioneered in recent years by Hong and colleagues, have shown that, in contrast, only a minor portion of xyloglucan interacts with cellulose ${ }^{8-14}$. In secondary cell walls, xylan (Supplementary Fig. 1) and glucomannan are known to bind to cellulose microfibrils, but there is little evidence for the mechanism of this interaction. As the main hemicellulose of eudicot secondary cell walls, the interaction of xylan with cellulose is expected to have the most profound effect on cell wall characteristics and therefore properties such as biomass recalcitrance and timber strength. Indeed, Arabidopsis plants with reduced xylan quantity show weakened walls and the plants are unable to develop a vascular system ${ }^{15,16}$. Molecular dynamics simulations suggest multiple layers of xylan could envelop microfibrils ${ }^{17}$, and reconstitution studies have revealed that xylan is able to crosslink microfibrils ${ }^{18}$, yet no clear model for the interaction has emerged. On the basis of a conserved pattern of xylan substitution strictly on alternate xylosyl residues, it has recently been proposed that xylan might be able to hydrogen bond to the hydrophilic surfaces of cellulose through folding as a twofold helical screw (Supplementary Fig. 2) ${ }^{19-22}$. This model is controversial because xylan in solution forms a threefold helical screw $^{19,23}$ (one $360^{\circ}$ twist per three glycosidic bonds). Despite the conservation of a periodic structure of xylan substitutions throughout seed plants, there is no direct experimental evidence for this model. MAS NMR has the potential to reveal polysaccharide conformations and interactions ${ }^{11,24-27}$. We have therefore recently developed its use to study Arabidopsis secondary cell wall ${ }^{28}$, so that mutants can be studied.

By combining Arabidopsis molecular genetics and MAS NMR approaches, here we show that xylan interacts intimately with cellulose by adopting a twofold helical screw conformation. The change of xylan fold is induced by, and dependent upon, cellulose fibrils. This provides the first direct experimental evidence for the atomic-scale arrangement of two distinct polymers within plant cell wall biomass, and reveals new principles underlying the remarkable specificity of polysaccharide interactions. Our work provides an understanding of the molecular architectural basis for the biological and mechanical function of cell wall components in plant biomass.

\section{Results}

Xylan adopts two distinct conformations in the cell wall. To analyse the structure of xylan in never-dried cell walls by ${ }^{13} \mathrm{C}$ MAS NMR, a chamber to grow uniformly labelled Arabidopsis plants in ${ }^{13} \mathrm{CO}_{2}$ was designed and constructed (Supplementary Fig. 3). Fresh and unprocessed stems were directly analysed, ensuring that the native arrangement of the cell wall polysaccharides was preserved. Figure la shows a two-dimensional (2D) refocused INADEQUATE ${ }^{25,29,30}$ MAS NMR spectrum of wild-type Arabidopsis stems using cross polarization $(\mathrm{CP})$ excitation, which emphasizes the more rigid components. In this experiment directly bonded nuclei appear at the same double-quantum (DQ) chemical shift that corresponds to the sum of the two single-quantum (SQ) chemical shifts. The spectrum shows broad line widths of $\sim 2$ p.p.m. indicating the relative rigidity of the polysaccharides and a range of different environments. As expected, the major peaks in the spectrum are from glucose in the two main cellulose environments $\left(C^{1}\right.$ : often assigned to crystalline and/or internal glucan chains; and $\mathrm{C}^{2}$ : often assigned to amorphous and/or surface glucan chains) ${ }^{8-12,28}$. Surprisingly, two distinct xylan carbon $4(\mathrm{Xn} 4)$ peaks are clearly visible in the spectrum (82.2 and 77.4 p.p.m.), each coupled to Xn5 (64.3 and 63.9 p.p.m.). The existence of the two discrete Xn4 shifts indicates the presence of two distinct conformations of xylan in the fresh hydrated cell walls. This is in contrast to the range of shifts observed for $\mathrm{Xn} 4$ in dried Arabidopsis stems ${ }^{28}$, suggesting that drying had a substantial effect on native xylan conformations.

Xylan in solution forms a threefold helical screw ${ }^{19,23}$. The 77.4 p.p.m. Xn4 and 63.9 p.p.m. Xn5 shifts are very similar to the solution state xylan Xn4 shift (Table 1), suggesting that they arise from threefold screw xylan in the cell wall, so we named them $\mathrm{Xn} 4^{3 \mathrm{f}}$ and $\mathrm{Xn} 5^{3 \mathrm{f}}$, respectively. It has been proposed that some xylan may fold as twofold screw in the cell wall ${ }^{19-22}$. A change in torsion angle around the glycosidic bond (which yields different helical conformations) can alter the chemical shifts of polysaccharides, and in $(1 \rightarrow 4)$-linked glycans changes in torsion angle has been shown to affect carbon 1 and carbon 4 resonances particularly ${ }^{27}$. The 82.2 p.p.m. Xn4 shift seen in the fresh cell walls might therefore arise from a change in conformation of the xylan to a twofold screw. To estimate the shift differences between the twofold screw and threefold screw xylan conformations, we performed in silico ${ }^{13} \mathrm{C}$ chemical shift prediction using gauge including projector augmented waves density functional theory $(\mathrm{DFT})^{31,32}$ (Fig. 1b). As expected, the two carbons involved in the glycosidic bond-Xn1 and Xn4-were predicted to alter the most after changing conformation (Table 1 and Supplementary Tables 1 and 2). $\mathrm{Xn} 1$ and Xn4 are predicted to be substantially shifted to higher p.p.m. in a twofold screw compared with a threefold screw conformation. Dominant cellulose signals mask the twofold $\mathrm{Xn} 1$ signal $\left(\mathrm{Xn}^{2 \mathrm{f}}\right)$ in the CP-refocused INADEQUATE spectrum (Fig. 1; Xn1 assigned below). However, the presence of the $\mathrm{Xn} 4$ signal at the higher (82.2 p.p.m., $\left.\mathrm{Xn} 4^{2 \mathrm{f}}\right){ }^{13} \mathrm{C}$ chemical shift, covalently linked to an Xn5 signal $\left(\mathrm{Xn} 5^{2 \mathrm{f}}\right)$, indicates that xylan forms a twofold screw in the cell wall.

Cellulose is required for xylan twofold screw conformation. Xylan might be induced to adopt the twofold screw conformation upon binding to cellulose in the cell wall ${ }^{19-22}$. Indeed, 82-84 p.p.m. peaks have previously been proposed to arise from cellulose-aggregated xylan ${ }^{33-36}$. Therefore, we next investigated whether the twofold screw xylan is dependent on cellulose in the cell wall. The irx3 Arabidopsis mutant is deficient in secondary 
a

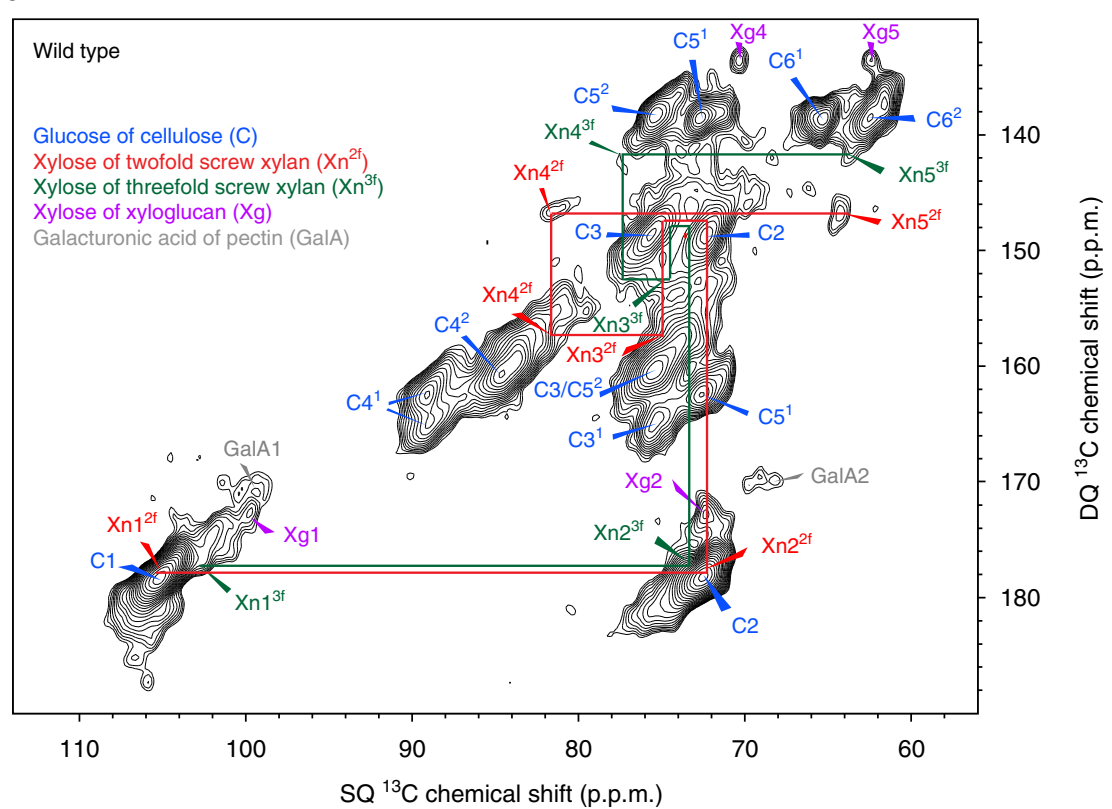

b
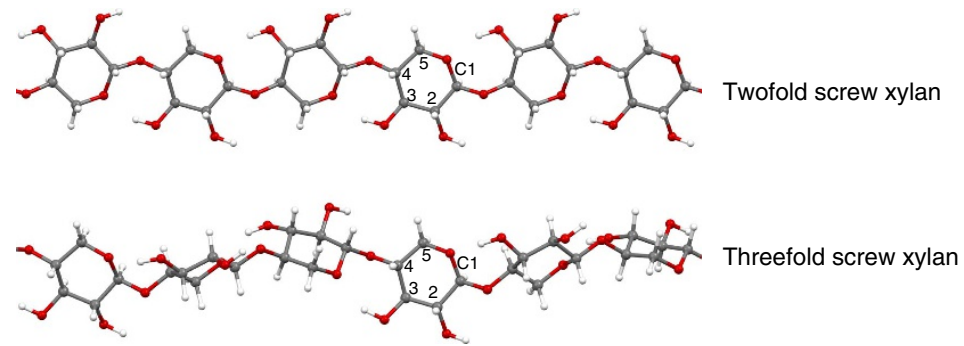

Figure 1 | Two distinct xylan populations in twofold screw and threefold screw helical conformations are found in never-dried wild-type Arabidopsis stems. (a) The carbohydrate region of a refocussed CP-INADEQUATE ${ }^{13} \mathrm{C}$ NMR spectrum of never-dried wild-type Arabidopsis stems is shown. Red and green labelling illustrates the two distinct xylan conformations, shown clearly here by the distinct xylan carbon 4 (Xn4) and Xn5 shifts. Xn1, Xn2 and Xn3 also differ between conformations but not all differences are readily distinguished in this CP spectrum (see Fig. 4 and Table 1 for full assignments). The spectrum was recorded at a ${ }^{13} \mathrm{C}$ Larmor frequency of $125.8 \mathrm{MHz}$ and a MAS frequency of $10 \mathrm{kHz}$ using a total spin-echo duration of $1.2 \mathrm{~ms}$. (b) In silico DFT-optimized xylan structures from which ${ }^{13} \mathrm{C}$ chemical shifts in Table 1 were calculated.

Table 1 | Comparison of observed and in silico predicted xylan ${ }^{13} \mathrm{C}$ chemical shifts.

\begin{tabular}{|c|c|c|c|c|c|}
\hline \multirow[b]{4}{*}{$\mathrm{Xn1}$} & \multicolumn{3}{|c|}{ Experimental ${ }^{13} \mathrm{C}$ chemical shifts (p.p.m.) } & \multicolumn{2}{|c|}{${ }^{13} \mathrm{C}$ chemical shift difference (p.p.m.) } \\
\hline & \multicolumn{2}{|c|}{ In cell wall } & \multirow{2}{*}{$\begin{array}{c}\text { In solution }{ }^{\star} \\
\text { Threefold }\end{array}$} & Observed in cell wall & Predicted from in silico ${ }^{\dagger}$ modelling \\
\hline & Twofold ${ }^{\ddagger}$ & Threefold ${ }^{\S}$ & & \multicolumn{2}{|c|}{ Twofold minus Threefold } \\
\hline & 105.2 & 102.6 & 102.5 & +2.6 & +4.0 \\
\hline$X n 2$ & 72.3 & 73.7 & 73.5 & -1.4 & -2.9 \\
\hline $\mathrm{Xn3}$ & (75.2) & 74.7 & 74.4 & $(+0.5)$ & +1.3 \\
\hline Xn4 & 82.2 & 77.4 & 77.2 & +4.8 & +8.2 \\
\hline $\mathrm{Xn} 5$ & 64.3 & 63.9 & 63.7 & +0.4 & +1.2 \\
\hline
\end{tabular}

cell wall cellulose synthesis and is consequently dwarfed, but the plant continues to synthesize other cell wall components including xylan ${ }^{37,38}$. Because it has much reduced secondary cell wall cellulose, it provides a useful tool to study the importance of cellulose for xylan conformation. One-dimensional MAS NMR of irx3 stems confirmed depletion in this mutant of cellulose and the relative increase in other cell wall polysaccharides, including xyloglucan $(\mathrm{Xg})$ and pectin (GalA) (Supplementary Fig. 4). Interestingly, a CP-refocused INADEQUATE spectrum showed a profound difference in the xylan conformation in irx3 compared with wild type. While twofold screw xylan ( $\mathrm{Xn} 4^{2 \mathrm{f}}$ peak) was by far the more 


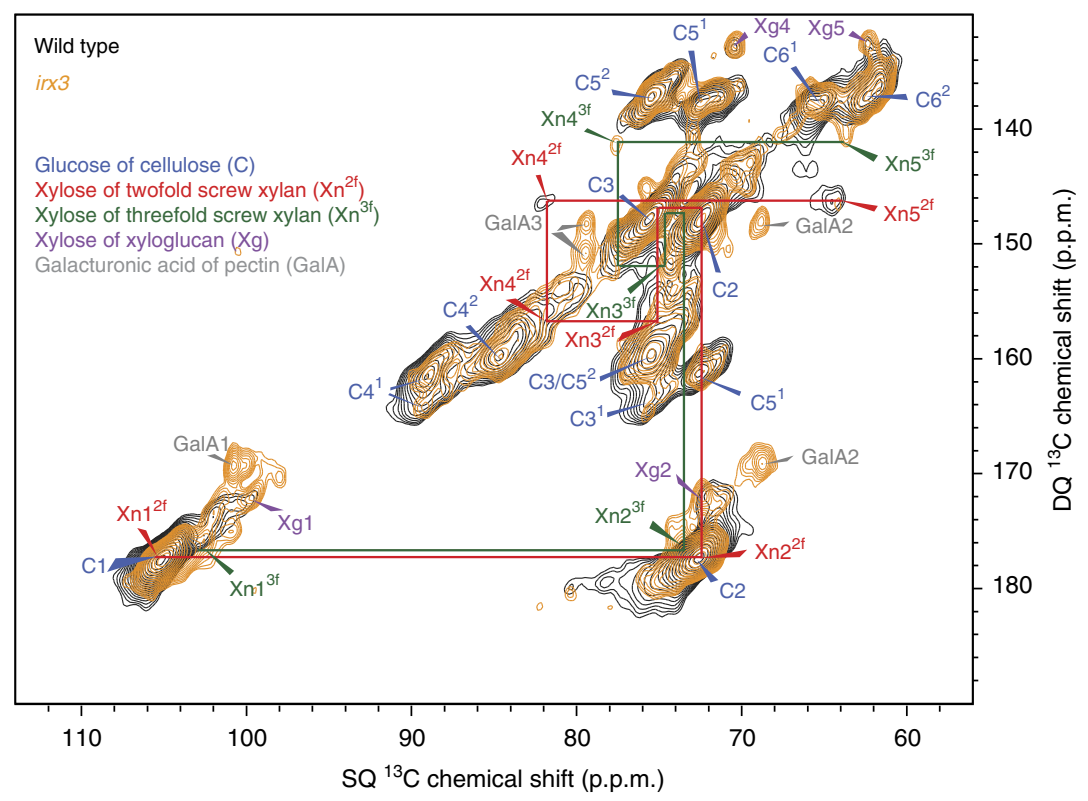

Figure 2 | Twofold screw xylan predominates in wild type but is barely detectable in the cellulose-deficient mutant irx3. An overlay is shown of carbohydrate regions of refocussed CP-INADEQUATE ${ }^{13} \mathrm{C}$ NMR spectra of wild-type and irx3. Red and green labelling illustrates the two distinct xylan conformations shown clearly here by the distinct $\mathrm{Xn} 4$ and $\mathrm{Xn} 5$ shifts. The spectra were recorded as in Fig. 1, using a total spin-echo duration of 2.2 ms.

prevalent of the two conformations in wild type (Figs 1 and 2), in irx3 it was barely detectable (Fig. 2). In contrast, almost all irx3 xylan was found in the threefold conformation. To study the more mobile cell wall components in irx3 and wild-type stems, direct polarization (DP)-INADEQUATE spectra with a short recycle delay were acquired. The abundance of the $\mathrm{Xn} 4^{3 \mathrm{f}}$ peak showed that relatively mobile threefold screw xylan is far more prevalent in irx3 than in the wild type (Fig. 3 and Supplementary Fig. 4). Indeed, this mobile threefold screw xylan was not detected in wild-type plants. Neither the twofold screw xylan nor cellulose was seen in the DP-INADEQUATE spectra of wild-type plants, indicating that the twofold screw xylan, like cellulose, is relatively immobile. The absence of the twofold screw xylan in irx3 shows that the change in xylan conformation from threefold to twofold screw is cellulose-dependent.

The presence of abundant threefold screw xylan in irx3 cell walls gave us the opportunity to investigate further the $\mathrm{Xn1}{ }^{2 \mathrm{f}}$ and $\mathrm{Xn} 1^{3 \mathrm{f}}{ }^{13} \mathrm{C}$ chemical shifts, given the DFT prediction of substantial change in Xn1 (Table 1). Using the knowledge that xylan is highly acetylated, the shifts of carbon atoms close to acetate moieties in the cell wall were investigated using protondriven spin diffusion (PDSD) experiments, which measure through-space proximities of different moieties ${ }^{39}$, with longer mixing times probing longer distances. Figure 4 shows a comparison of long mixing time PDSD spectra of wild-type plants (a CP spectrum where mainly twofold xylan is visible), with irx3 (a DP-PDSD spectrum where only threefold xylan is visible). Acetate methyl groups (21.6 p.p.m.) are spatially close to acetate carbonyl groups (173.6 p.p.m.) and also to Xn1-Xn5. Interestingly, the Xn1 ${ }^{31}$ shift in irx3 is 102.6 p.p.m. as seen in DP INADEQUATE and in solution xylan, but in wild-type $\mathrm{Xn} 1^{2 \mathrm{f}}$ is significantly shifted to the higher ${ }^{13} \mathrm{C}$ chemical shift of 105.2 p.p.m.. Because a clear increase in $\mathrm{Xn}^{2 \mathrm{f}}$ shift from threefold to twofold was predicted by the calculations (Table 1), this observation further supports the assignment of the conformation which predominates in wild type as twofold screw, and the conformation which predominates in irx3 as threefold. Notably, the $\mathrm{Xn} 1^{2 \mathrm{f}}$ shift of 105.2 p.p.m. is very similar to cellulose $\mathrm{C} 1$ at 105.1 p.p.m., which also exists in a twofold screw.

Xylan with a twofold screw conformation is bound to cellulose. To determine whether the twofold screw xylan is cellulose-bound, spatial proximities of molecules in the cell wall were investigated using a relaxation compensated $z$-filtered version of the CP-PDSD experiment ${ }^{40}$ (Fig. 5). In the experiment with a short $50 \mathrm{~ms}$ mixing time, cross peaks indicative of short-distance intramolecular spatial relationships were observed, for example, within cellulose domain 1 (C4 ${ }^{1}-\mathrm{C} 6^{1} ; 89.0$ p.p.m.-65.3 p.p.m.), cellulose domain $2\left(\mathrm{C}^{2}-\mathrm{C}^{2} ; 84.1\right.$ p.p.m. to 62.6 p.p.m.) and twofold screw xylan (Xn4 ${ }^{2 \mathrm{f}}-\mathrm{Xn} 5^{2 \mathrm{f}} ; 82.2$ p.p.m. to 64.2 p.p.m.; Supplementary Table 1). In the experiment with a longer, $1 \mathrm{~s}$, mixing time cross peaks indicative of both short and longer distance intra- and intermolecular spatial relationships were observed. Interestingly, cross peaks were seen between xylan and cellulose, indicating their close spatial proximity. However, xylan cross peaks to cellulose domain 1 (for example, $\mathrm{Xn} 4^{2 \mathrm{f}} \rightarrow \mathrm{C} 6^{1}$; Fig. $5 c, d$ ), which is often assigned to internal cellulose glucan chains, were greater than those to cellulose domain 2 (for example, $\mathrm{Xn} 4^{2 \mathrm{f}} \rightarrow \mathrm{C}^{2}$ Fig. $5 \mathrm{c}$ ), which is often assigned to surface cellulose glucans. This suggests that xylan coating of cellulose surface chains causes a change in glucan NMR shift, perhaps because these glucans adopt an interior-like conformation, akin to a recent proposal for cellulose: hemicellulose interactions in primary cell walls ${ }^{13}$. The similar signal strengths of the intermolecular $\mathrm{Xn}^{2 \mathrm{f}} \rightarrow \mathrm{C}^{1}$ and the intramolecular $\mathrm{Xn} 4^{2 \mathrm{f}} \rightarrow \mathrm{Xn} 5^{2 \mathrm{f}}$ peaks indicate that almost all of the twofold screw xylan is spatially close to cellulose. In contrast at this mixing time the cross peaks showing spatial proximities between cellulose domains (for example, $\mathrm{C} 4^{1} \rightarrow \mathrm{C}^{2}$ and $\mathrm{C}^{1} \rightarrow \mathrm{C}^{2}$ ) are not yet as strong as those showing spatial proximities within cellulose domains (for example, $\mathrm{C} 4^{1} \rightarrow \mathrm{C} 6^{1}$ ). Although spin diffusion in a fully ${ }^{13} \mathrm{C}$ labelled system is complex, 


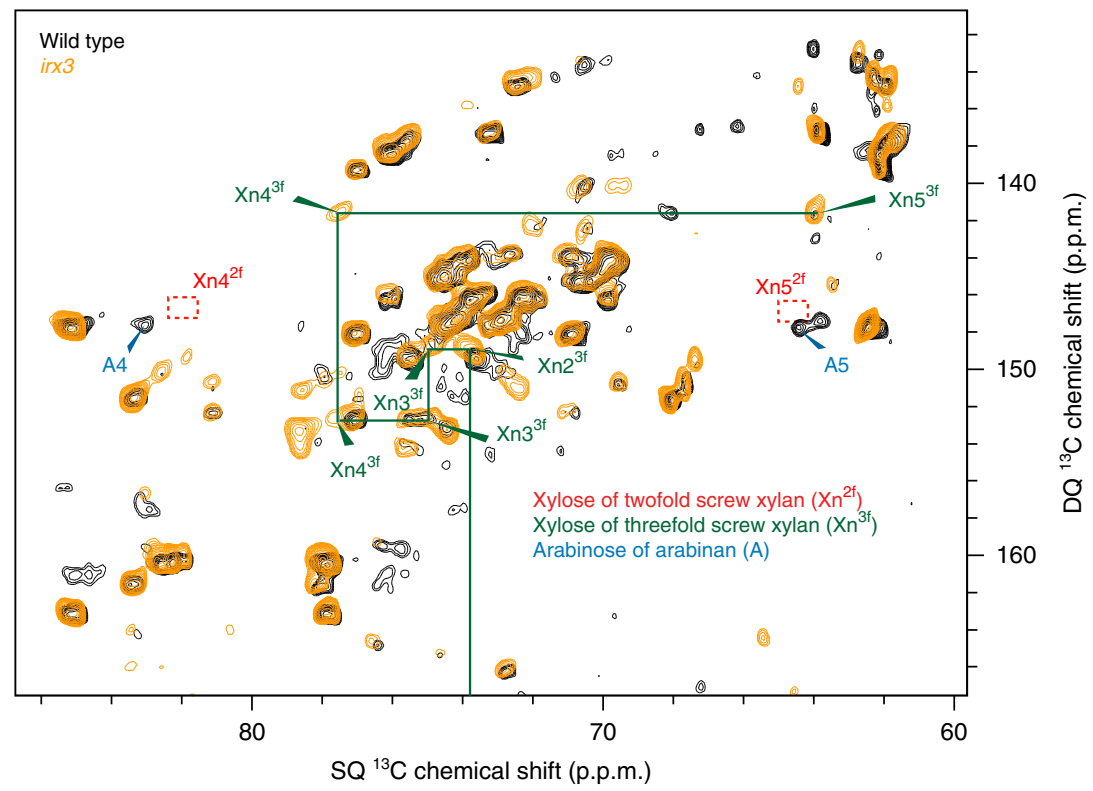

Figure 3 | Threefold screw xylan is abundant in irx3 but not in wild type as shown by analysis of mobile cell wall components. An overlay is shown of ${ }^{13} \mathrm{C}$ refocussed DP-INADEQUATE ${ }^{13} \mathrm{C}$ NMR spectra of wild type and irx3 never-dried Arabidopsis stems using a $1.9 \mathrm{~s}$ recycle time, which emphasize relatively mobile species. The carbohydrate region of the spectrum is expanded to focus on the $\mathrm{Xn} 4 \rightarrow \mathrm{Xn} 5$ connection. This shows that threefold xylan is abundant in irx 3 , and the twofold xylan is relatively immobile and so is not detected in wild type or irx3 mutant cell walls. Xylose of threefold xylan is highlighted in green. Dotted red squares indicate the absence of the twofold screw $\mathrm{Xn} 4 \rightarrow \mathrm{Xn} 5$ pair. The change in some arabinose and pectin structures in the plants may be due to altered growth of the dwarfed irx3 plant. The spectrum of the full carbohydrate region is shown in Supplementary Figure 5, and was recorded with a spin-echo duration of $2.2 \mathrm{~ms}$ at a ${ }^{13} \mathrm{C}$ Larmor frequency of $213.8 \mathrm{MHz}$ and a MAS frequency of $12 \mathrm{kHz}$.

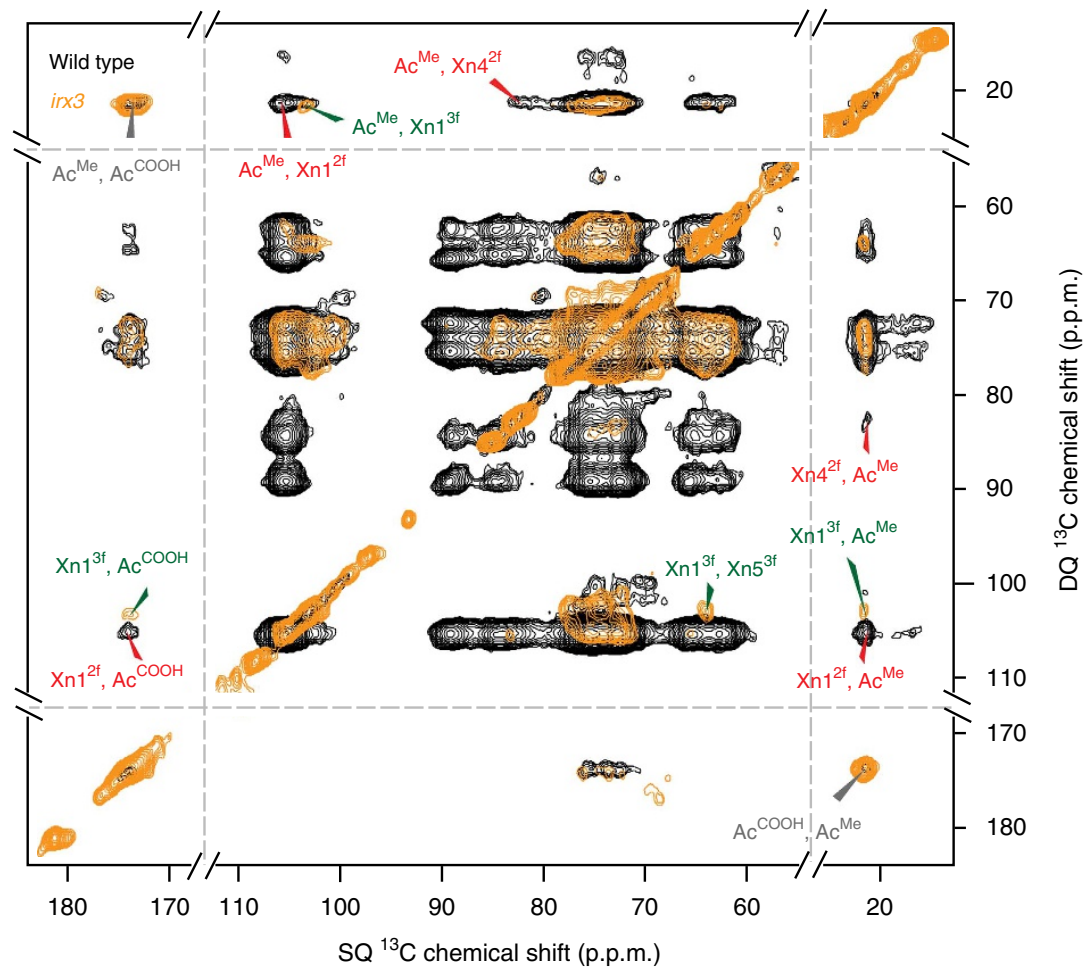

Figure 4 | Twofold and threefold screw xylan have distinct carbon 1 shifts. An overlay is shown of ${ }^{13} \mathrm{C}$ PDSD NMR spectra of wild type (CP-PDSD $1 \mathrm{~s}$ mixing time) and irx3 (DP-PDSD, $1.5 \mathrm{~s}$ mixing time) never-dried Arabidopsis stems. Wild-type and irx3 show two distinct xylan conformations: in wild-type plants $\mathrm{Xn1}^{2 \mathrm{ff}}$ resonates at 105.2 p.p.m., whereas in $i r \times 3 \mathrm{Xn1}^{3 \mathrm{f}}$ is at 102.6 p.p.m. These long mixing time spectra were recorded at a ${ }^{13} \mathrm{C}$ Larmor frequency of 213.8 $\mathrm{MHz}$ and a MAS frequency of $12 \mathrm{kHz}$. 

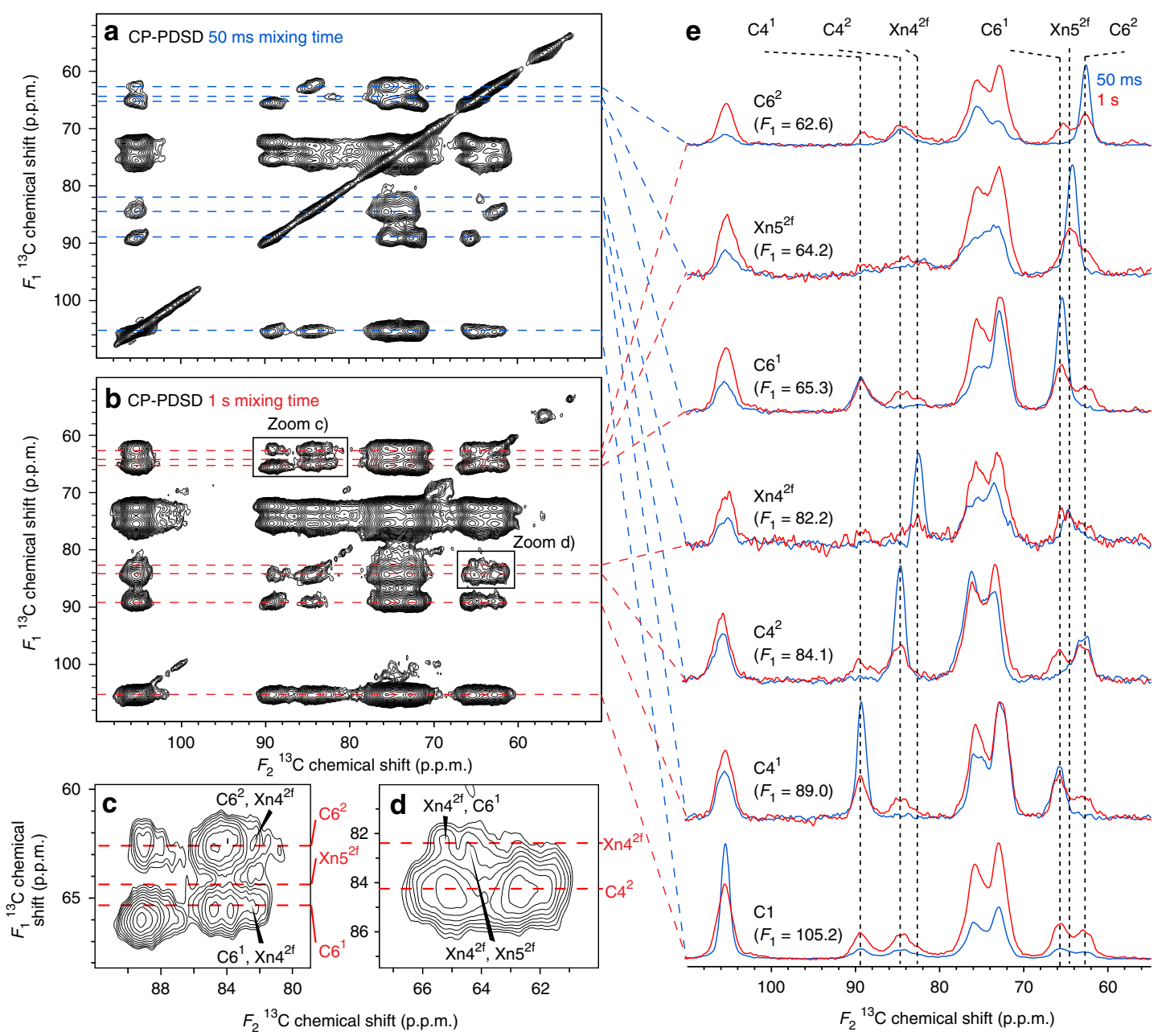

Figure 5 | Twofold screw xylan is found in close proximity to cellulose in never-dried wild-type Arabidopsis stems. Carbohydrate regions are shown of $z$-filtered CP-PDSD ${ }^{39}{ }^{13} \mathrm{C}$ NMR spectra of never-dried wild-type Arabidopsis stems using a total z period of $1.005 \mathrm{~s}$. (a) $50 \mathrm{~ms}$ mixing time spectrum. (b) $1 \mathrm{~s}$ mixing time spectrum. (c) Zoom of $1 \mathrm{~s}$ mixing time spectrum showing cellulose $\rightarrow$ xylan connections. (d) Zoom of $1 \mathrm{~s}$ mixing time spectrum showing xylan $\rightarrow$ cellulose connections. (e) One-dimensional slices from $50 \mathrm{~ms}$ (blue) and 1s (red) mixing time spectra. The spectra were recorded as in Fig. 4.

the presence of the relatively large $\mathrm{Xn} 4^{2 \mathrm{f}} \rightarrow \mathrm{C}^{1}$ cross peak means that we can place an upper limit on the distance between the twofold screw xylan and cellulose: twofold screw xylan can be no farther than the width of a microfibril from the cellulose surface. Hence, the PDSD experiments indicate that the twofold screw xylan is bound to cellulose.

As a further means of probing xylan: cellulose interactions we analysed the molecular mobility of these polysaccharides by measuring the ${ }^{13} \mathrm{C} \quad T_{1}$ relaxation time (Supplementary Table 3), which is sensitive to motion on the nanosecond timescale, and the dipolar order parameter $S_{C H}\left(0 \leq S_{C H} \leq 1\right)$ which is sensitive to motion on the microsecond timescale. Both DP and CP DIPSHIFT ${ }^{41}$ experiments were used to measure the order parameter as there is more than one contribution to the signal for many chemical shifts. The DP DIPSHIFT with $20 \mathrm{~s}$ recycle delay was quantitative with all of the species contributing, whereas the CP DIPSHIFT preferentially measured the immobile components such as cellulose and any cellulose-bound xylan. Figure 6 shows a comparison of the $\mathrm{CP}$ order parameters representative of cellulose, twofold xylan and threefold xylan, all obtained from the dephasing curves shown in Supplementary Fig. 6, for both wild-type and irx3 samples. Cellulose-bound xylan would be expected to exhibit less motion (demonstrated by a larger order parameter) than unbound xylan. The order parameter of twofold xylan (specifically measuring $\mathrm{Xn} 4^{2 \mathrm{f}}$ in wildtype stems) is comparable to that of both cellulose domains (specifically measuring $\mathrm{C} 4^{1}$ and $\mathrm{C}^{2}$ in wild-type stems), indicating relatively little motion. In contrast, for threefold xylan (specifically measuring $\mathrm{Xn} 1^{3 \mathrm{f}}$ and $\mathrm{Xn} 4^{3 \mathrm{f}}$ in $i r \times 3$ stems) the order parameter is significantly reduced, indicating more motion. Consistent with this, the $T_{1}$ of $X n 4^{2 f}(82.5$ p.p.m.) in wild type is very similar to cellulose values whereas $\mathrm{Xn} 1^{3 \mathrm{f}}$ in ir $x 3$ has shorter $T_{1}$ (see Supplementary Table 3). Therefore, twofold screw xylan is far less mobile than threefold screw xylan, and has a similar rigidity to cellulose.

The CP-INADEQUATE and DP-INADEQUATE experiments together indicated that the large majority of xylan in the secondary cell walls of wild-type plants folds as twofold screw, with only a minor proportion of xylan detected in a relatively immobile threefold screw conformation. The PDSD and DIPSHIFT experiments, together with the requirement for cellulose revealed by $i r \times 3$, indicated that essentially all the twofold screw xylan is bound to cellulose. These results are consistent with the finding that the majority of xylan has substitution patterning that is compatible with binding to the hydrophilic surfaces of cellulose $e^{19-21}$. However, we do not yet know the relative amounts of the xylan bound to the 


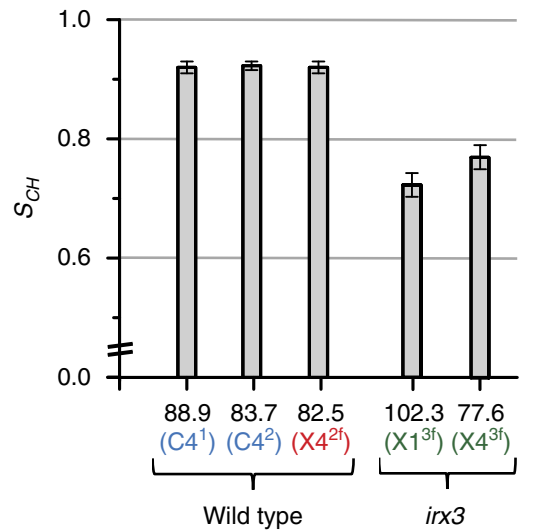

Figure 6 | Cellulose and twofold screw xylan are similarly rigid, while threefold screw xylan is more mobile. CP-DIPSHIFT MAS NMR analysis of the order parameter, $\mathrm{S}_{\mathrm{CH}}$, in wild-type and irx3 stems is shown; this reports on the molecular mobilities of cell wall moieties. The labelling refers to the presumed major contributing species. Refer to Supplementary Fig. 4 for chemical shift assignments of the One-dimensional spectra. See Supplementary Fig. 6 for data fits. The error bars were determined by simulation of the DIPSHIFT curves shown in Supplementary Fig. $6 \mathrm{~b}$ with varying parameters. The error bar represents the maximum change in parameter before there is a clear deviation of simulated from experimental values. The order parameters for all major peaks are shown in Supplementary Fig. 7. Experiments were carried out at a ${ }^{13} \mathrm{C}$ Larmor frequency of $125.8 \mathrm{MHz}$ and a MAS frequency of $7.813 \mathrm{kHz}$.

hydrophilic vs hydrophobic surfaces of the fibrils. Moreover, the proportion of hydrophilic and hydrophobic surfaces on the cellulose microfibril structure is not yet known. In the secondary cell walls of eudicots, the ratio between quantity of xylan and cellulose is approximately 2:3 (ref. 42). Our results therefore suggest that there is likely sufficient xylan to coat the hydrophilic surfaces of cellulose microfibrils. Nevertheless, further work is necessary to determine the extent of xylan binding to the different fibril surfaces.

\section{Discussion}

The molecular arrangement of plant cell wall components has been an unresolved question for decades. We have now shown that xylan is induced to assemble on cellulose fibrils as a twofold helical screw in secondary cell walls. This is the first direct evidence for the atomic-scale model of the interactions between cellulose and xylan in plant cell walls (Supplementary Fig. 2) ${ }^{19,20}$. In this model, xylan hydrogen bonds with cellulose microfibril hydrophilic surfaces by forming a flattened twofold helical screw. This effectively extends the semi-crystalline cellulose microfibril size and alters its properties. Coating of the hydrophilic faces of cellulose microfibrils with the acetylated and glucuronosylated form of xylan found in dicot cell walls may lead the otherwise hydrophilic surfaces to be relatively hydrophobic (because of acetate groups) and acidic (because of glucuronic acid groups). This may be of profound importance to cell wall architecture, for example influencing interactions between the cellulose-xylan fibrils, and altering binding to the other components of the secondary cell wall, especially lignin. Xylan folded onto cellulose surfaces is likely to be resistant to the action of microbial hydrolases which cleave threefold screw xylan. It remains unknown whether some xylan may also bind to the hydrophobic surface of fibrils. The evenly spaced glucuronosyl and acetyl substitutions of xylan ${ }^{19-22}$ are important for allowing xylan to bind to the hydrophilic surfaces of cellulose. Because xylan from gymnosperms to eudicots has retained this even pattern of substitution ${ }^{22}$, we believe the assembly of xylan onto cellulose revealed in this work is likely to be conserved across land plants. Through discovery of the specific interaction of these two polysaccharides, this work therefore reveals a fundamental principle in assembly of plant cell walls which likely extends to other polysaccharide interactions and the extracellular matrix of many organisms.

A description of the nature of the interactions between cell wall components is crucial for many uses of plants as a renewable resource. It helps us understand the basis of plant recalcitrance to digestibility and deconstruction, which is of importance in dietary fibre, animal fodder and in biofuel production. These findings will also be important in improving processes using materials made from plants, such as the paper industry and building construction. Our studies of cell wall molecular architecture therefore provide a new twist for exploitation of plant materials.

\section{Methods}

Growth chamber and plant preparation. irx3-7 seeds were kindly donated by Simon Turner (University of Manchester). Seeds were surface sterilized and sown on $0.8 \%(\mathrm{w} / \mathrm{v})$ agar, 0.59 Murashige and Skoog salts including vitamins (Sigma, http://www.sigma.com) and sucrose (1\% w/v). Following stratification for $48 \mathrm{~h}$ at $4{ }^{\circ} \mathrm{C}$ in the dark, plates were transferred to a growth room $\left(20^{\circ} \mathrm{C}, 100 \mu \mathrm{mol} \mathrm{m}^{-2} \mathrm{~s}^{-1}, 24 \mathrm{~h}\right.$ light, $60 \%$ humidity). Optimaxx fibre rockwool (Cultilene, Netherlands) was cut into slabs and laid $\sim 8 \mathrm{~cm}$ deep in a growth tray. Hydroponics solution $\left(2 \mathrm{mM} \mathrm{MgSO}_{4}, 2 \mathrm{mM} \mathrm{CaNO}_{3}, 50 \mu \mathrm{M}\right.$ FeEDTA, $5 \mathrm{mM}$ $\mathrm{KNO}_{3}, 2.5 \mathrm{mM} \mathrm{K}_{2} \mathrm{HPO}_{4} / \mathrm{KH}_{2} \mathrm{PO}_{4} \mathrm{pH} 5.5,70 \mu \mathrm{M} \mathrm{H}_{3} \mathrm{BO}_{4}, 14 \mu \mathrm{M} \mathrm{MnCl}, 0.5 \mu \mathrm{M}$ $\mathrm{CuSO}_{4}, 1 \mu \mathrm{M} \mathrm{ZnSO}_{4}, 0.2 \mu \mathrm{M} \mathrm{NaMoO}, 10 \mu \mathrm{M} \mathrm{NaCl}$ and $0.1 \mu \mathrm{M} \mathrm{CoCl}_{2}$ ) was poured into the tray until half-way up. The rockwool was then covered with foil. Holes were pierced into the foil and rockwool and seedling were placed in, ensuring that roots made direct contact with rockwool. A growth chamber was constructed following Chen et al. ${ }^{43}$ illustrated in Supplementary Fig. 3. Compressed air was scrubbed of $\mathrm{CO}_{2}$ using calcium oxide before ${ }^{13} \mathrm{CO}_{2}$ was mixed back in at a concentration of 500 p.p.m. before entering the growth chamber. Plants were grown in a $50 \%$ humidity and $24{ }^{\circ} \mathrm{C}$ environment for $6-8$ weeks. ${ }^{13} \mathrm{C}$ enrichment was measured by analysing xyloglucan oligosaccharides yielded from xyloglucan endoglucanase digestion of stems using matrix-assisted laser desorption/ ionization-time of flight mass spectrometry. Typical enrichments were 90-95\%. Roughly $35 \mathrm{mg}$ of the bottom third parts of five to ten stems were chopped and packed into a $3.2 \mathrm{~mm}$ Magic-Angle Spinning NMR rotor. For wild-type, five biological replicates (of at least five plants) were grown, each of which was analysed at least once; for irx3, two biological replicates (of at least five plants) were grown, each of which was analysed at least once. On the basis of the relative content of cellulose, pectin, xyloglucan and xylan observed in the wild-type and irx3 samples by NMR, we estimate the stem material contained a substantial but minority proportion of primary cell walls.

NMR chemical shift predictions. The NMR shift calculations were carried out using the CASTEP code ${ }^{44}$ with the $\mathrm{PBE}^{45}$ exchange correlation functional. The 10 molecule (two- and threefold) MD xylan structures generated earlier ${ }^{19}$ were first geometry optimized using a cutoff energy of $800 \mathrm{eV}$ with a $k$ point grid of $2 \times 2 \times 1$ and a fixed unit cell of $10 \times 10 \times 60 \AA$. The NMR shielding was calculated for the resulting structures using the gauge including projector augmented waves ${ }^{31,32}$ method. The reference used to convert shielding to shift, 167.7 p.p.m., was determined by assuming that the threefold structure is very similar to xylan in solution (see Supplementary Table 2). Its value does not affect the shift differences given in Table 1 .

Solid-state NMR. Solid-state MAS NMR experiments were performed on Bruker (Karlsruhe, Germany) 850 and $500 \mathrm{MHz}$ Advance III solid-state NMR spectrometers, operating at ${ }^{1} \mathrm{H}$ and ${ }^{13} \mathrm{C}$ Larmor frequencies of 850.2 and $213.8 \mathrm{MHz}$ and 500.1 and $125.8 \mathrm{MHz}$, respectively, using $3.2 \mathrm{~mm}$ double-resonance MAS probes. Experiments were conducted at room temperature at MAS frequencies of $12-14 \mathrm{kHz}$ at the higher field and $10 \mathrm{kHz}$ on the $500 \mathrm{MHz}$ spectrometer unless otherwise stated. The ${ }^{13} \mathrm{C}$ chemical shift was determined using the carbonyl peak at 177.8 p.p.m. of L-alanine as an external reference with respect to tetramethylsilane (TMS); $90^{\circ}$ pulse lengths were typically $3.5 \mu \mathrm{s}\left({ }^{1} \mathrm{H}\right)$ and $4.2 \mu \mathrm{s}\left({ }^{13} \mathrm{C}\right)$. Both ${ }^{1} \mathrm{H}-{ }^{13} \mathrm{C}$ cross-polarization $(\mathrm{CP})$ with ramped $(70-100 \%)$ ${ }^{1} \mathrm{H}$ rf amplitude ${ }^{46}$ and a contact time of $1 \mathrm{~ms}$ and direct polarization (DP) were used to obtain the initial transverse magnetization. While CP emphasizes the more rigid material, a short, $1.9 \mathrm{~s}$, recycle delay DP experiment was used to preferentially detect the mobile components and a $20 \mathrm{~s}$ delay was used for 
quantitative experiments. SPINAL-64 decoupling ${ }^{47}$ was applied during acquisition at a ${ }^{1} \mathrm{H}$ nutation frequency of $70-80 \mathrm{kHz}$. Two-dimensional double-quantum (DQ) correlation spectra were recorded using the refocused INADEQUATE pulse sequence which relies upon the use of isotropic, scalar J coupling to obtain through-bond information regarding directly coupled nuclei ${ }^{25,29,30}$. The carbon $90^{\circ}$ and $180^{\circ}$ pulse lengths were 4 and $8 \mu$ s, respectively with $2 \tau$ spin-echo evolution times for a $(\tau-\pi-\tau)$ spin-echo of 1.2 to $4.4 \mathrm{~ms}$ and the SPINAL-64 ${ }^{1} \mathrm{H}$ decoupling ${ }^{47}$ was applied during both the evolution and signal acquisition periods. The acquisition time in the indirect dimension $\left(t_{1}\right)$ was $4.5-5.0 \mathrm{~ms}$ with a recycle delay of 1.5 and $1.9 \mathrm{~s}$ for $\mathrm{CP}$ and DP experiments, respectively. Intermolecular contacts were probed using $2 \mathrm{D}^{13} \mathrm{C}-{ }^{13} \mathrm{C}{ }^{1} \mathrm{H}$ driven spin diffusion (PDSD) experiments ${ }^{39,40}$ with mixing times of $50 \mathrm{~ms}$ to $1.5 \mathrm{~s}$. For both refocused INADEQUATE and PDSD experiments, the spectra were obtained by Fourier transformation into $2 \mathrm{~K}(\mathrm{~F} 2) \times 1 \mathrm{~K}\left(F_{1}\right)$ points with exponential line broadening of $80 \mathrm{~Hz}(\mathrm{CP})$ and $40 \mathrm{~Hz}(\mathrm{DP})$ in $F_{2}$ and squared sine bell processing in $F_{1}$. The ${ }^{13} \mathrm{C}$ spin lattice relaxation time, $T_{1}$, was measured at $125.8 \mathrm{MHz}$ using saturation recovery following a comb of 30 pulses and echo acquisition. The dipolar order parameter, $S_{C H}$, was determined using the $2 \mathrm{D}^{13} \mathrm{C}-{ }^{1} \mathrm{H}$ dipolar chemical shift (DIPSHIFT) experiment ${ }^{41}$ whilst spinning at $7.813 \mathrm{kHz}$ on the $500 \mathrm{MHz}$ spectrometer. The ${ }^{1} \mathrm{H}$ homonuclear coupling was suppressed using the frequency switched Lee-Goldberg sequence ${ }^{48}$ with a ${ }^{1} \mathrm{H}$ nutation frequency of $80 \mathrm{kHz}$. Measurements were taken with CP and with DP excitation with recycle delays of 2 and $20 \mathrm{~s}$, respectively. All spectra obtained were processed and analysed using Bruker Topspin version 3.2.

DIPSHIFT calculations. DIPSHIFT experiments were simulated using a home written program in Origin.c. The DIPSHIFT curves were calculated as a simple evolution of the ${ }^{13} \mathrm{C}$ magnetization under $\mathrm{C}-\mathrm{H}$ dipolar coupling and MAS. The effective $\mathrm{C}-\mathrm{H}$ coupling for $\mathrm{CH}_{2}$ units was obtained using the expressions reported by Terao et al. ${ }^{49}$ This provides a reliable measurement of the effective $\mathrm{C}-\mathrm{H}$ coupling in the fast and rigid motion limits, as confirmed by comparison with full spin dynamics simulations ${ }^{50}$.

Data availability. The solid-state NMR data and scripts for DIPSHIFT simulations are available at http://dx.doi.org/10.17863/CAM.5896. The authors declare that all other relevant data supporting the findings of this study are available within the article and its Supplementary Information files or on request from the corresponding authors.

\section{References}

1. Perlack, R. D. \& Stokes, B. J., Leads. U.S. Billion-Ton Update: Biomass Supply for a Bioenergy and Bioproducts Industry. Technical Report ORNL/TM-2011/ 224, 227. (U.S. Department of Energy, Oak Ridge National Laboratory, Oak Ridge, TN, 2011).

2. Carpita, N. \& McCann, M. in Biochemistry and Molecular Biology of Plants, Vol. 61 (eds Buchanan, B. B., Wilhelm, G., and Jones, R. L.) 52-108, (American Society of Plant Physiologists, Rockville, IL, 2000).

3. Scheller, H. V. \& Ulvskov, P. Hemicelluloses. Annu. Rev. Plant Biol. 61, 263-289 (2010).

4. Atalla, R. H. \& Vanderhart, D. L. Native cellulose - a composite of 2 distinct crystalline forms. Science 223, 283-285 (1984).

5. Nishiyama, Y., Langan, P. \& Chanzy, H. Crystal structure and hydrogenbonding system in cellulose $1 \beta$ from synchrotron X-ray and neutron fiber diffraction. J. Am. Chem. Soc. 124, 9074-9082 (2002).

6. Nishiyama, Y., Sugiyama, J., Chanzy, H. \& Langan, P. Crystal structure and hydrogen bonding system in cellulose $1 \alpha$, from synchrotron X-ray and neutron fiber diffraction. J. Am. Chem. Soc. 125, 14300-14306 (2003).

7. Cosgrove, D. J. \& Jarvis, M. C. Comparative structure and biomechanics of plant primary and secondary cell walls. Front. Plant Sci. 3, 1-6 (2012).

8. Dick-Perez, M. et al. Structure and Interactions of plant cell-wall polysaccharides by two- and three-dimensional magic-angle-spinning solidstate NMR. Biochemistry 50, 989-1000 (2011).

9. Dick-Perez, M., Wang, T., Salazar, A., Zabotina, O. A. \& Hong, M. Multidimensional solid-state NMR studies of the structure and dynamics of pectic polysaccharides in uniformly C-13-labeled Arabidopsis primary cell walls. Magn. Reson. Chem. 50, 539-550 (2012).

10. Wang, T., Zabotina, O. \& Hong, M. Pectin-cellulose interactions in the arabidopsis primary cell wall from two-dimensional magic-angle-spinning solid-state nuclear magnetic resonance. Biochemistry 51, 9846-9856 (2012).

11. Wang, T. et al. Sensitivity-enhanced solid-state NMR detection of expansin's target in plant cell walls. Proc. Natl Acad. Sci. USA 110, 16444-16449 (2013).

12. Wang, T., Salazar, A., Zabotina, O. A. \& Hong, M. Structure and dynamics of brachypodium primary cell wall polysaccharides from two-dimensional ${ }^{13} \mathrm{C}$ solid-state nuclear magnetic resonance spectroscopy. Biochemistry $\mathbf{5 3}$, 2840-2854 (2014).

13. Wang, T., Yang, H., Kubicki, J. D. \& Hong, M. Cellulose structural polymorphism in plant primary cell walls investigated by high-field $2 \mathrm{D}$
Solid-state NMR spectroscopy and density functional theory calculations. Biomacromolecules 17, 2210-2222 (2016).

14. Bootten, T. J., Harris, P. J., Melton, L. D. \& Newman, R. H. Solid-state ${ }^{13} \mathrm{C}$-NMR spectroscopy shows that the xyloglucans in the primary cell walls of mung bean (Vigna radiata L.) occur in different domains: a new model for xyloglucan-cellulose interactions in the cell wall. J. Exp. Bot. 55, 571-583 (2004).

15. Wu, A. M. et al. The Arabidopsis IRX10 and IRX10-LIKE glycosyltransferases are critical for glucuronoxylan biosynthesis during secondary cell wall formation. Plant J. 57, 718-731 (2009).

16. Brown, D. M. et al. Comparison of five xylan synthesis mutants reveals new insight into the mechanisms of xylan synthesis. Plant J. 52, 1154-1168 (2007).

17. Li, L., Perre, P., Frank, X. \& Mazeau, K. A coarse-grain force-field for xylan and its interaction with cellulose. Carbohyd. Polym. 127, 438-450 (2015).

18. Mikkelsen, D., Flanagan, B. M., Wilson, S. M., Bacic, A. \& Gidley, M. J. Interactions of arabinoxylan and $(1,3)(1,4)-\beta$-glucan with cellulose networks. Biomacromolecules 16, 1232-1239 (2015).

19. Busse-Wicher, M. et al. The pattern of xylan acetylation suggests xylan may interact with cellulose microfibrils as a twofold helical screw in the secondary plant cell wall of Arabidopsis thaliana. Plant J. 79, 492-506 (2014).

20. Busse-Wicher, M., Grantham, N. J., Lyczakowski, J. J., Nikolovski, N. \& Dupree, P. Xylan decoration patterns and the plant secondary cell wall molecular architecture. Biochem. Soc. Trans. 44, 74-78 (2016).

21. Bromley, J. R. et al. GUX1 and GUX2 glucuronyltransferases decorate distinct domains of glucuronoxylan with different substitution patterns. Plant J. 74, 423-434 (2013).

22. Busse-Wicher, M. et al. Evolution of xylan substitution patterns in gymnosperms and angiosperms: implications for xylan interaction with cellulose. Plant Physiol. 171, 2418-2431 (2016).

23. Nieduszynski, I. \& Marchessault, R. H. Structure of $\beta$-D- $\left(1 \rightarrow 4^{\prime}\right)$ Xylan Hydrate. Nature 232, 1335-1344 (1971).

24. Bardet, M., Emsley, L. \& Vincendon, M. Two-dimensional spin-exchange solidstate NMR studies of ${ }^{13} \mathrm{C}$-enriched wood. Solid State Nucl. Magn. Reson. 8, 25-32 (1997).

25. Lesage, A., Bardet, M. \& Emsley, L. Through-bond carbon-carbon connectivities in disordered solids by NMR. J. Am. Chem. Soc. 121, 10987-10993 (1999).

26. Komatsu, T. \& Kikuchi, J. Selective signal detection in solid-state NMR Using rotor-synchronized dipolar dephasing for the analysis of hemicellulose in lignocellulosic biomass. J. Phys. Chem. Lett. 4, 2279-2283 (2013).

27. Jarvis, M. C. \& Apperley, D. C. Chain conformation in concentrated pectic gels - evidence from ${ }^{13} \mathrm{C}$ NMR. Carbohyd. Res. 275, 131-145 (1995).

28. Dupree, R. et al. Probing the molecular architecture of Arabidopsis thaliana secondary cell walls using two- and three-dimensional ${ }^{13} \mathrm{C}$ solid state nuclear magnetic resonance spectroscopy. Biochemistry 54, 2335-2345 (2015).

29. Lesage, A., Auger, C., Caldarelli, S. \& Emsley, L. Determination of throughbond carbon-carbon connectivities in solid-state NMR using the INADEQUATE experiment. J. Am. Chem. Soc. 119, 7867-7868 (1997).

30. Fayon, F. et al. Through-space contributions to two-dimensional doublequantum J correlation NMR spectra of magic-angle-spinning solids. J. Chem. Phys. 122, 14931 (2005).

31. Pickard, C. J. \& Mauri, F. All-electron magnetic response with pseudopotentials: NMR chemical shifts. Phys. Rev. B 63, 245101 (2001).

32. Yates, J. R., Pickard, C. J. \& Mauri, F. Calculation of NMR chemical shifts for extended systems using ultrasoft pseudopotentials. Phys. Rev. B 76, 024401 (2007).

33. Wickholm, K., Larsson, P. T. \& Iversen, T. Assignment of non-crystalline forms in cellulose I by CP/MAS ${ }^{13} \mathrm{C}$ NMR spectroscopy. Carbohyd. Res. 312, 123-129 (1998).

34. Larsson, P. T., Hult, E. L., Wickholm, K., Pettersson, E. \& Iversen, T. CP/MAS C-13-NMR spectroscopy applied to structure and interaction studies on cellulose I. Solid State Nucl. Magn. Reson. 15, 31-40 (1999).

35. Teleman, A., Larsson, P. T. \& Iversen, T. On the accessibility and structure of xylan in birch kraft pulp. Cellulose 8, 209-215 (2001).

36. Habibi, Y., Heux, L., Mahrouz, M. \& Vignon, M. R. Morphological and structural study of seed pericarp of Opuntia ficus-indica prickly pear fruits. Carbohyd. Polym. 72, 102-112 (2008).

37. Ha, M. A. et al. Structure of cellulose-deficient secondary cell walls from the irx3 mutant of Arabidopsis thaliana. Phytochemistry 61, 7-14 (2002).

38. Brown, D. M., Zeef, L. A. H., Ellis, J., Goodacre, R. \& Turner, S. R. Identification of novel genes in Arabidopsis involved in secondary cell wall formation using expression profiling and reverse genetics. Plant Cell 17, 2281-2295 (2005).

39. Takegoshi, K., Nakamura, S. \& Terao, T. ${ }^{13} \mathrm{C}-{ }^{1} \mathrm{H}$ dipolar-assisted rotational resonance in magic-angle spinning NMR. Chem. Phys. Lett. 344, 631-637 (2001). 
40. Wang, T., Williams, J. K., Schmidt-Rohr, K. \& Hong, M. Relaxationcompensated difference spin diffusion NMR for detecting ${ }^{13} \mathrm{C}-{ }^{13} \mathrm{C}$ long-range correlations in proteins and polysaccharides. J. Biomol. NMR 61, 97-107 (2015).

41. Munowitz, M. G., Griffin, R. G., Bodenhausen, G. \& Huang, T. H. Two-dimensional rotational spin-echo nuclear magnetic-resonance in solids correlation of chemical-shift and dipolar interactions. J. Am. Chem. Soc. 103, 2529-2533 (1981).

42. Pauly, M. \& Keegstra, K. Cell-wall carbohydrates and their modification as a resource for biofuels. Plant J. 54, 559-568 (2008).

43. Chen, W. P. et al. An automated growth enclosure for metabolic labeling of Arabidopsis thaliana with ${ }^{13} \mathrm{C}$-carbon dioxide - an in vivo labeling system for proteomics and metabolomics research. Proteome Sci. 9, 9 (2011).

44. Clark, S. J. et al. First principles methods using CASTEP. Z. Kristallogr 220, 567-570 (2005).

45. Perdew, J. P., Burke, K. \& Ernzerhof, M. Generalized gradient approximation made simple. Phys. Rev. Lett. 77, 3865-3868 (1996).

46. Metz, G., Wu, X. L. \& Smith, S. O. Ramped-amplitude cross-polarization in magic-angle-spinning NMR. J. Magn. Reson. Ser. A 110, 219-227 (1994).

47. Fung, B. M., Khitrin, A. K. \& Ermolaev, K. An improved broadband decoupling sequence for liquid crystals and solids. J. Magn. Reson. 142, 97-101 (2000).

48. Bielecki, A., Kolbert, A. C. \& Levitt, M. H. Frequency-switched pulse sequences - homonuclear decoupling and dilute spin NMR in solids. Chem. Phys. Lett. 155, 341-346 (1989).

49. Terao, T., Miura, H. \& Saika, A. Dipolar SASS NMR-spectroscopy - separation of heteronuclear dipolar powder patterns in rotating solids. J. Chem. Phys. 85, 3816-3826 (1986).

50. DeAzevedo, E. R. et al. Intermediate motions as studied by solid-state separated local field NMR experiments. J. Chem. Phys. 128, 128-139 (2008).

\section{Acknowledgements}

We thank Dinu Iuga, Shazeaa Ishmael, Howard Griffiths and Moritz Meyer for their help. This work was part supported by BBSRC Grant BB/G016240/1 via The BBSRC Sustainable Bioenergy Cell Wall Sugars Programme. ODB and ERdA are grateful to CNPq for financial support for this work via Grants \# 159341/2011-6 and 206278/2014-4. ACP is grateful to the Royal Society for a Newton International Fellowship. PD is supported by the Leverhulme Trust grant for the Centre for Natural Material Innovation. The UK $850 \mathrm{MHz}$ solid-state NMR Facility used in this research was funded by EPSRC and BBSRC, as well as the University of Warwick including via part funding through Birmingham Science City Advanced Materials Projects 1 and 2 supported by Advantage West Midlands (AWM) and the European Regional Development Fund (ERDF). (Contract reference PR140003 for work after 5 January 2015). DFT calculations of NMR parameters were performed at the Centre for Scientific Computing at the University of Warwick.

\section{Author contributions}

T.J.S. grew plants. J.C.M. designed and constructed the growth chamber. R.D., O.B. and T.J.S. conducted the NMR experiments. T.J.S., J.C.M., O.B., S.P.B., E.A., R.D. and P.D. analysed the experimental data. A.C.P. performed in silico NMR chemical shift predictions. T.J.S., R.D. and P.D. wrote the manuscript. All authors contributed critical thoughts.

\section{Additional information}

Supplementary Information accompanies this paper at http://www.nature.com/ naturecommunications

Competing financial interests: The authors declare no competing financial interests.

Reprints and permission information is available online at http://npg.nature.com/ reprintsandpermissions/

How to cite this article: Simmons, T. J. et al. Folding of xylan onto cellulose fibrils in plant cell walls revealed by solid-state NMR. Nat. Commun. 7, 13902 doi: 10.1038/ncomms13902 (2016).

Publisher's note: Springer Nature remains neutral with regard to jurisdictional claims in published maps and institutional affiliations.

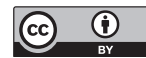

This work is licensed under a Creative Commons Attribution 4.0 International License. The images or other third party material in this article are included in the article's Creative Commons license, unless indicated otherwise in the credit line; if the material is not included under the Creative Commons license, users will need to obtain permission from the license holder to reproduce the material. To view a copy of this license, visit http://creativecommons.org/licenses/by/4.0/

(C) The Author(s) 2016 